\title{
Niveles plasmáticos de citoquinas IL-1ß, IL2 e IL-4 en niños diabéticos tipo 1 de diagnóstico reciente y su asociación con anticuerpos ß pancreáticos
}

\author{
Francisco Pérez $B^{1 a}$, Amaya 0 yarzún $A^{1 b}$, Elena Carrasco $P^{2 c}$, \\ Bárbara Angel B ${ }^{1 d}$, Cecilia Albala $B^{1}$, J Luis Santos $M^{12}$. \\ Plasma levels of interleukin-1ß, \\ interleukin-2 and interleukin-4 in recently \\ diagnosed type 1 diabetic children and \\ their association with B-pancreatic \\ autoantibodies
}

Background: Type 1 diabetes is an organ specifc autoimmune disease whose incidence is increasing worldwide. A functional imbalance in cytokine production resulting in dominance of T helper (Th1) over Th2-type response has been suggested to play a critical role in the pathogenesis of type 1 diabetes. Aim: To measure serum concentrations of interleukin (IL)-1ß, IL-2 and IL-4 in children with recently diagnosed type 1 diabetes and to evaluate the autoimmune response measuring glutamic acid decarboxylase (GAD65) and tyrosine phosphatase like (IA-2) autoantibodies. Patients and Methods: 120 diabetic children and 118 age and gender matched control children, were recruited for this study. Circulating levels of IL-1ß, IL-2 and IL-4 were measured by ELISA. GAD65 and IA-2 were measured by RIA. Results: Circulating levels of IL-1ß were elevated in type 1 diabetic children as compared to the control group $(9.3 \pm 7.3$ and $4.9 \pm 3.8 \mathrm{pg} / \mathrm{ml}$ respectively, $\mathrm{p}=0,01)$. Serum concentration of IL-2 was also higher in diabetic patients (19.8 \pm 13.1 and $11.3 \pm 9.1 \mathrm{pg} / \mathrm{ml}$ respectively, $p=0,01$ ). No differences in serum IL-4 were observed between diabetics and control. Diabetic children with one or two positive autoantibodies (IA-2 and/or GAD65) had significantly higher levels of IL-1ß and IL-2 and lower levels of IL-4 than diabetic children without positive autoantibodies. High concentrations of IL-1ß were associated with an early onset of the disease. Conclusions: High levels of IL-1ß and IL-2 were found in diabetic children with recent diagnosis of the disease. Diabetics with positive antibodies against GAD65 and IA-2 had higher levels of IL-1ß and IL-2 and lower levels of IL-4 than their counterparts without positive antibodies (Rev Méd Chile 2004; 132: 413-20).

(Key Words: Diabetes mellitus, insulin dependent; Interleukin-1; Interleukin-2; Interleukin-4)

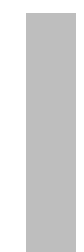

\footnotetext{
Recibido el 28 de enero, 2003. Aceptado en versión corregida el 6 de enero, 2004.

Trabajo financiado íntegramente por intermedio de los proyectos FONDECYT \#1000944 y FONDECYT \#1030680.

${ }^{1}$ Programa de Epidemiología Genética. Instituto de Nutrición y Tecnología de los Alimentos (INTA), Universidad de Chile. ${ }^{2}$ Unidad de Diabetes, Hospital San Juan de Dios. Facultad de Medicina, Universidad de Chile. Santiago de Chile.

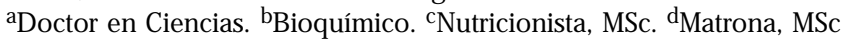

Correspondencia a: Dr. Francisco Pérez-Bravo. Programa

de Epidemiología Genética, INTA-Universidad de Chile.

Casilla 138-11, Santiago. E mail: fperez@inta.cl 
L a diabetes tipo 1 es una enfermedad multifactorial, de etiología heterogénea, en la cual, en la mayoría de los casos, la célula beta pancreática es destruida por un complejo mecanismo inmune, determinado principalmente por la actividad del complejo mayor de histocompatibilidad (MHC) y en forma particular, por algunas moléculas del sistema HLA, genes DRB1 y DQ en clase II $^{1}$. En la diabetes tipo 1, los padres transmiten a sus hijos una predisposición para adquirir la enfermedad en algún momento de la vida ${ }^{1,2}$.

En la última década, las investigaciones inmunológicas acerca de la herencia y los eventuales mecanismos de autoagresión involucrados en la diabetes tipo 1, así como la implicancia de diversos factores ambientales (virus, toxinas $\mathrm{y}$ dieta), han revolucionado el concepto de la enfermedad y plantean nuevas estrategias terapéuticas para el futuro ${ }^{3,4}$.

No se conoce con certeza el mecanismo responsable de la destrucción de las células $ß$ pancreáticas, pero existe amplia evidencia que relaciona la respuesta autoinmune, con una serie de eventos celulares, tales como la infiltración celular en el islote, la aparición de autoanticuerpos (entre ellos: ICAt, GAD + o IA-2+) y una alteración del equilibrio inmunológico Th1/Th2 $2^{5,6}$.

En la diabetes tipo 1 se puede describir una alteración central y periférica en la tolerancia de la célula $\beta$ frente a determinados antígenos. El desequilibrio Th1/Th2 es un proceso evidente durante el fenómeno de la insulitis ${ }^{7}$. A nivel de mecanismo, son las moléculas HLA de clase II las capacitadas para señalizar al linfocito la inducción de determinadas citoquinas. Dentro de las respuestas Th1, es posible observar una inducción de IL-1ß, IL-2, INFy, moléculas que poseen actividad pleiotrópica sobre la célula ß. La respuesta Th1 es facilitadora del complejo celular, mientras que la respuesta Th2 (IL-4, IL-10, etc.) favorece la respuesta de tipo citotóxica ${ }^{8-10}$.

Las citoquinas son, en resumen, potentes mediadores del sistema inmune a nivel de presentación antigénica. IL-1ß fue la primera citoquina que, incubada en islotes, produjo la inhibición en la secreción insulínica postestímulo de glucosa ${ }^{11}$. Otras citoquinas como TNF $\alpha$ INFr e IL-2, elaboradas por el macrófago y células dendríticas generan quimiotaxis, estimulan linfocitos, sobreexpresan moléculas de adhesión y estimulan al macrófago residente en el islote a la producción de IL-1/ ${ }^{11,12}$.

Dentro de las citoquinas inmunosupresoras, la IL-4 y la IL-10 han sido utilizadas, con resultados variables, en diferentes modelos animales como posibles señales protectoras de diabetes tipo 1. En el ratón NOD, la sobreexpresión de IL-10 reduce los niveles de INFy e IL-12, retardando la aparición del cuadro diabético. Particularmente IL-4, ha generado un amplio debate y controversia en relación al posible papel protector que tendría esta citoquina en la aparición y progresión de la enfermedad, dado que existe una serie de antecedentes que indican lo contrario ${ }^{13,23}$.

Considerando que existe amplia evidencia relacionada a un desbalance Th1/Th2 en la diabetes tipo 1, el propósito de este estudio ha sido analizar los niveles circulantes de tres citoquinas ampliamente descritas en la patogenia de la enfermedad (IL-1ß, IL-2 e IL-4) en un grupo de niños debutantes con diabetes tipo 1 y en un grupo de niños controles. Del mismo modo, evaluar si existe asociación entre una mayor agresividad $ß$ pancreática (mediada por altos títulos de autoanticuerpos GAD65 e IA-2) y estas citoquinas.

\section{PACIENTES Y MÉTODOS}

Los casos incidentes de diabetes de tipo 1 se obtuvieron de centros asistenciales públicos y privados. La primera fuente de información de casos pesquisados, correspondió a cada Unidad de Diabetes de los hospitales y clínicas participantes y como fuente secundaria de recolección de los casos, se utilizó la información de los registros de la Fundación de Diabetes Juvenil de Chile. Esta investigación incluye los casos nuevos diagnosticados en la Región Metropolitana durante el período comprendido entre marzo del año 1999 hasta octubre del año 2002, menores de 15 años, que aceptaron participar. A todos ellos se les entregó información respecto de los objetivos y proyecciones del proyecto (Fondecyt \#1000944) y se les solicitó un consentimiento escrito de participación voluntaria. El grupo de estudio estuvo constituido por 120 niños diabéticos, con una edad promedio de $8,5 \pm 3,5$ años (66 hombres y 54 
mujeres). Además, se consideró un grupo control de niños $(n=118)$ con rangos de edad comparables $(9,1 \pm 5,5$ años, 62 hombres y 56 mujeres), situación socioeconómica similar, sin antecedentes familiares de diabetes tipo $1 \mathrm{u}$ otras patologías autoinmunes, provenientes de 3 colegios de Santiago (sector sur y centro).

\section{Metodología}

En todos los casos incidentes y controles se recolectó una única muestra de $5 \mathrm{ml}$ de sangre para la obtención de suero y plasma. En el momento de la toma de muestra, al menos uno de los padres debió estar presente para firmar la autorización correspondiente. En todos los casos se aplicó una encuesta para recopilar la información necesaria sobre antecedentes familiares de otras enfermedades, especialmente aquellas de origen autoinmune.

La obtención de la muestra de sangre en los casos con diabetes tipo 1 , fue realizada en un período comprendido entre 0 y 2 semanas posterior al debut. En general, la mayoría de los casos fue pesquisado durante el período de estabilización en el estadio inicial de la enfermedad, mientras el niño se encontraba internado. Se incluyen todos los niños de debut reciente, sin antecedente de cetoacidosis. La muestra de sangre obtenida luego de la punción venosa, fue procesada en el Laboratorio de Epidemiología Genética del INTA, centrifugada a $2.500 \mathrm{rpm}$ en centrifuga convencional durante $10 \mathrm{~min}$. El plasma y suero obtenidos, fueron colectados en tubos Eppendorf de $1,5 \mathrm{ml}$, alicuotado en $100 \mu \mathrm{l}$ y congelado a $-80^{\circ} \mathrm{C}$ hasta la realización de las diversas determinaciones de anticuerpos y citoquinas. Para la determinación de anticuerpos anti-decarboxilasa del ácido glutámico (GAD) y anticuerpos antitirosina fosfatasa (IA-2) se empleó un radioinmunoanálisis (RIA) de los Laboratorios Cis Bio International (Francia). Ambos sistemas utilizan GAD e IA-2 humana recombinante, emplean como trazador ${ }^{125} \mathrm{I}$ y permiten establecer un sistema de discriminación de positividad o negatividad frente a la curva de calibración. El coeficiente de variación intraensayo para anti-GAD fue de 2,9\% y de 3,1\% para anti-IA-2. El coeficiente de variación interensayo fue de 3,6\% para anti-GAD y de 5,1\% para anti-IA-2. El perfil de citoquinas IL1ß, IL-2 e IL-4 se realizó utilizando el sistema de ELISA de los laboratorios Roche (Alemania), con un coeficiente de variación promedio intraensayo de $2,3 \%$ e interensayo de $3,1 \%$.

La validación de los datos y su análisis fue realizado con el programa estadístico STATA 7.0. Los valores se expresaron como media y desviación estándar. Para realizar las comparaciones entre los grupos se utilizaron las pruebas de (») Student o Mann-Whitney.

\section{RESULTADOS}

La Tabla 1 resume la información relacionada a la concentración de citoquinas en niños diabéticos y controles según sexo y grupos de edad. Se observaron diferencias significativas cuando se compararon los grupos totales, estando IL-1ß aumentada, tanto en los niños diabéticos $(p=0,006)$, como en las niñas diabéticas $(p=0,001)$ en relación a niños y niñas del grupo control respectivamente. Una situación similar se observó para la concentración de IL-2, la cual estuvo incrementada en niños y niñas diabéticas respecto a sus respectivos grupos controles $(p=0,001)$. IL-2 fue la única citoquina que mostró diferencias cuando se realizaron comparaciones entre niños y niñas de cada grupo, observándose un incremento de esta citoquina en los niños diabéticos respecto a las niñas diabéticas en el grupo de edad comprendido entre 5-9 años $(p=0,031)$. En relación a IL-4 no se observaron diferencias significativas entre niños y niñas con y sin diabetes, ni diferencias al comparar los grupos totales.

La Tabla 2 muestra la concentración sérica de estas citoquinas, de acuerdo al perfil de autoinmunidad ß pancreática mediada a través de GAD65 e IA-2 en el grupo de niños diabéticos. La concentración de las tres citoquinas determinadas no fue diferente al comparar los grupos con la presencia de 1 ó 2 anticuerpos positivos. Sin embargo, la concentración sérica de IL-1ß e IL-2 fue significativamente menor en aquellos niños diabéticos que no presentaron títulos positivos para estos 2 autoanticuerpos ( $p<0,001$ en ambos casos). Por otro lado, aquellos diabéticos con títulos negativos 
Tabla 1. Concentración de citoquinas IL-1ß, IL-2 e IL-4 en diabéticos y controles según sexo y grupos de edad

\begin{tabular}{|c|c|c|c|c|c|c|}
\hline & \multicolumn{2}{|c|}{ IL-1ß (pg/ml) } & \multicolumn{2}{|c|}{ IL-2 (pg/ml) } & \multicolumn{2}{|c|}{$\mathrm{IL}-4(\mathrm{pg} / \mathrm{ml})$} \\
\hline & Niños & Niñas & Niños & Niñas & Niños & Niñas \\
\hline \multicolumn{7}{|l|}{ Casos $(n=120)$} \\
\hline 0-4 años & $9,4 \pm 7,5$ & $11,8 \pm 10,1$ & $24,2 \pm 11,8$ & $21,7 \pm 11,7$ & $7,5 \pm 5,3$ & $7,9 \pm 4,1$ \\
\hline 5-9 años & $8,8 \pm 7,0$ & $5,5 \pm 4,6$ & $24,3 \pm 17,3$ & $13,1 \pm 10,3^{*}$ & $8,1 \pm 7,5$ & $10,0 \pm 4,1$ \\
\hline 10-14 años & $8,3 \pm 6,9$ & $12,1 \pm 11,0$ & $16,7 \pm 13,1$ & $22,3 \pm 14,6$ & $6,9 \pm 3,8$ & $9,6 \pm 6,6$ \\
\hline Total & $8,7 \pm 7,2^{\#}$ & $10,1 \pm 7,4^{\mathrm{x}}$ & $20,5 \pm 14,9^{x}$ & $18,9 \pm 12,9^{\mathrm{x}}$ & $7,4 \pm 5,6$ & $9,2 \pm 5,7$ \\
\hline \multicolumn{7}{|c|}{ Controles $(\mathrm{n}=118)$} \\
\hline 0-4 años & $9,3 \pm 7,3$ & $3,6 \pm 2,3$ & $7,6 \pm 3,9$ & $10,4 \pm 5,8$ & $9,3 \pm 6,2$ & $7,7 \pm 3,7$ \\
\hline 5-9 años & $5,1 \pm 2,9$ & $4,9 \pm 3,1$ & $11,9 \pm 9,8$ & $9,3 \pm 7,3$ & $9,4 \pm 7,6$ & $10,5 \pm 6,1$ \\
\hline 10-14 años & $3,9 \pm 2,7$ & $4,6 \pm 2,1$ & $11,9 \pm 9,5$ & $16,1 \pm 11,8$ & $8,9 \pm 3,7$ & $7,6 \pm 4,3$ \\
\hline Total & $5,3 \pm 4,9^{\#}$ & $4,6 \pm 2,7^{x}$ & $11,2 \pm 9,5^{x}$ & $11,4 \pm 8,8^{x}$ & $9,2 \pm 6,4$ & $9,0 \pm 5,3$ \\
\hline
\end{tabular}

${ }^{*} p=0,031 \quad$ (niños versus niñas en el grupo diabético para el rango 5-9 años).

$\#_{p}=0,006 \quad$ (IL-1ß niños diabéticos versus niños controles).

$x_{p}=0,001 \quad$ (IL-1ß niñas diabéticas versus niñas controles).

(IL-2 niños diabéticos versus niños controles).

(IL-2 niñas diabéticas versus niñas controles).

Tabla 2. Concentración de citoquinas IL-1ß, IL-2 e IL-4 de acuerdo a la presencia de autoanticuerpos ß pancreáticos $\mathrm{GAD}_{65}$ e IA-2 en niños diabéticos recién diagnosticados

\begin{tabular}{|lccc|}
\hline & $\begin{array}{c}2 \text { anticuerpos } \\
\text { positivos } \\
\left(\mathrm{GAD}_{65} \text { e IA-2) }\right. \\
(\mathrm{n}=43)\end{array}$ & $\begin{array}{c}1 \text { anticuerpo } \\
\text { positivo } \\
\left(\mathrm{GAD}_{65} \text { o IA-2 }\right) \\
(\mathrm{n}=52)\end{array}$ & $\begin{array}{c}2 \text { anticuerpos } \\
\text { negativos } \\
\left(\mathrm{GAD}_{65} \text { e IA-2) }\right. \\
(\mathrm{n}=25)\end{array}$ \\
\hline $\mathrm{IL}-1 \Omega(\mathrm{pg} / \mathrm{ml})$ & $9,9 \pm 3,1$ & $8,4 \pm 3,9$ & $4,7 \pm 2,1^{*}$ \\
$\mathrm{IL}-2(\mathrm{pg} / \mathrm{ml})$ & $24,9 \pm 8,4$ & $26,1 \pm 9,2$ & $13,2 \pm 6,2^{*}$ \\
$\mathrm{IL}-4(\mathrm{pg} / \mathrm{ml})$ & $6,8 \pm 3,5$ & $6,3 \pm 2,9$ & $10,9 \pm 2,1^{*}$ \\
\hline
\end{tabular}

$* p<0,01$ perfil negativo versus presencia de 1 ó 2 anticuerpos positivos.

IA-2 y GAD65 mostraron niveles significativamente mayores de IL-4 ( $p<0,001)$.

Finalmente, en relación a la edad del diagnóstico, aquellos niños con bajos niveles de IL-1ß (2$5 \mathrm{pg} / \mathrm{ml}$ ) presentaron un debut más tardío, a una edad promedio de 9,3 $\pm 3,1$ años, cifra estadísticamente diferente a aquellos niños con altos niveles de esta citoquina, quienes debutaron en promedio a los $6,8 \pm 3,8$ años $(p<0,009)$. No se observó ninguna correlación de este tipo al comparar la edad al debut con los niveles de IL-2 o IL-4.

\section{DISCUSIÓN}

La liberación de citoquinas y la movilización de linfocitos desde las células presentadoras de antígenos, parecen tener un papel clave en la modulación de la respuesta autoinmune que media la destrucción de la célula beta pancreática ${ }^{8}$.

Existe una clara evidencia que demuestra una alteración en el patrón de distribución de ciertas citoquinas en los pacientes diabéticos, sin embargo no hay un consenso absoluto respecto a cuáles 
citoquinas representan el mejor indicador que se correlacione con el daño pancreático ${ }^{7,8}$. Dentro de los estudios en humanos, como en aquellos realizados en los modelos animales, se ha podido apreciar que la IL-1ß corresponde a una de las citoquinas que mayor efecto ejerce a nivel pancreático, principalmente reduciendo la producción de insulina. Por el contrario, IL-4 e IL-5 parecen ser las citoquinas que muestran un mayor efecto sobre la producción de preproinsulina en ciertos cultivos celulares ${ }^{8-12}$.

Nuestros datos muestran una importante variabilidad en la concentración de las citoquinas IL-1ß e IL-2, al comparar pacientes recién diagnosticados con niños controles, siendo este un fenómeno ya descrito por otros estudios ${ }^{10-13}$. Por otra parte, se observó que la IL-4, citoquina asociada controversialmente con protección contra la diabetes tipo 1, no varió significativamente al comparar a los niños controles respecto al grupo diabético.

Existe claridad en la literatura en relación a que los mayores niveles de IL-1ß e IL-2 se asociarían a una respuesta autoinmune incrementada, pero no se ha descrito ningún mecanismo que pueda dar cuenta de esta relación. Nuestro estudio, sólo encontró asociación entre altos títulos de IL-1ß y menor edad al debut, situación no observada para IL-2 o IL-4. Tampoco se observaron diferencias importantes en la concentración de estas citoquinas según sexo para los rangos de edad preestablecidos.

Son diversos los estudios que relacionan variabilidad en los niveles de ciertas citoquinas con susceptibilidad al desarrollo de diabetes tipo 1 . Investigaciones de los últimos años han mostrado evidencias contradictorias respecto del potencial rol de la IL-4 como posible target» para terapia génica en la diabetes tipo $1^{8}$. Existe una serie de trabajos que indican que el uso de plasmidios recombinantes (por ejemplo GAD65-IgGfC-IL-4) podrían tener un eventual papel protector ${ }^{14-20}$. Sin embargo, también hay fuerte evidencia respecto a lo inapropiado que puede resultar un posible enfoque terapéutico con esta citoquina ${ }^{21-23}$.

En relación al perfil de anticuerpos GAD65 e IA-2, datos de nuestro laboratorio publicados en el año 200124, reflejaron que ambos anticuerpos se distribuyen en forma diferente entre los diabéticos con períodos variables de lactancia materna.
En este sentido, es reconocido que la lactancia materna efectiva, potencia claramente la disminución de ciertas citoquinas alergenas y podría, en este caso, tener alguna relación con el perfil de autoinmunidad más agresivo que se presenta en algunos niños que comparten un alto título de IL$1 ß$ y un reducido período de lactancia materna ${ }^{25}$.

Respecto a otras citoquinas asociadas a la patogenia de la diabetes tipo 1 , recientemente se ha demostrado que IL-1ß, TNF $\alpha$ e IFN $\gamma$ son capaces de ejercer un fuerte papel inhibitorio sobre la expresión del autoantígeno IA-2 ${ }^{26}$. Por otra parte, IL-8 ha sido asociada a diagnósticos de niños con diabetes tipo 1 poseedores de altos índices de masa corporal, probablemente por una sobreproducción de IL-8 adipocitaria, capaz de desestabilizar el circuito inmunorregulador a nivel pancreático ${ }^{13}$.

Recientemente, se ha planteado el papel potencial que tendría IL-12ß como blanco de prevención de la diabetes tipo 1. El locus genético de IL-12ß ha mostrado una fuerte asociación con los clásicos HLA clase II de riesgo. Lo más novedoso de esta citoquina, está dado por el hecho que sus niveles de expresión responden rápidamente a determinadas intervenciones de la dieta, entre ellas el contenido de PUFA, vitaminas liposolubles y consumo de alcohol ${ }^{27}$. Tanto los PUFA como los complejos vitamínicos liposolubles, son capaces de reducir los niveles de IL-12ß probablemente

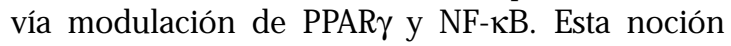
surge, dado que elementos de NF- $\kappa B$ han sido encontrados en el promotor de IL-12ß. En modelos animales que reciben suplementos de vitamina A, D3 y E, se reducen significativamente los niveles de IL-12ß y la expresión de su ARN mensajero, reduciéndose claramente la incidencia de diabetes tipo 1 en estos modelos animales condicionados genéticamente al desarrollo de diabetes $^{27}$.

Resulta importante destacar que, en todo estudio que involucre la cuantificación de citoquinas plasmáticas, existe un gran número de factores críticos que pueden alterar su concentración en forma temporal y que, por lo tanto, pueden inducir a una interpretación equivocada. En nuestro estudio, se excluyeron del análisis final aquellos pacientes que debutaron con cetoacidosis, puesto que existen informes en la literatura que 
indican que la inestabilidad metabólica del paciente diabético debutante altera el patrón serológico de ciertas citoquinas ${ }^{13,28}$.

Resulta evidente considerar, que cualquier componente asociado a infección recurrente, también alterará la concentración de las citoquinas circulantes. $\mathrm{Si}$ bien es un aspecto difícil de controlar, una de las formas de selección de nuestras muestras séricas, fue la consideración de proteína $C$ reactiva negativa.

Nuestro análisis también excluyó de su tamaño muestral original (tanto en casos como en controles), niños con diagnóstico dudoso de diabetes tipo 2 y niños con obesidad. Desde el punto de vista metabólico, el exceso de tejido adiposo produce concentraciones elevadas en determinadas citoquinas (características de un proceso de adipogénesis aumentada) tales como la IL-6, IL-8 y $\mathrm{TNF}^{13,28}$. En estos casos, un desbalance de cualquiera de ellas es capaz de modificar el equilibrio Th1/Th2, generando un patrón de distribución distinto, el cual no tendría relación con un mecanismo inmunológico clásico, sino más bien sería una alteración generada por el exceso de tejido adiposo. En este sentido, la acumulación de grasa abdominal en el paciente diabético tipo 1 , se asocia además a un pobre control metabólico, a mayores necesidades insulínicas y a un perfil lipídico alterado ${ }^{29}$.

Existe controversia respecto del mecanismo por el cual estas citoquinas generadas por el tejido adiposo serían capaces de desestabilizar a la célula $ß$ pancreática, sin embargo, evidencias preliminares indican que dichas citoquinas inducirían la sobreexpresión de Fas en la célula beta, evento independiente de la presencia o no de susceptibilidad genética a la enferme$\operatorname{dad}^{29,30}$.

Aún no existe consenso para plantear un mecanismo único que relacione el patrón de citoquinas con la destrucción del islote pancreático, sin embargo, desde el planteamiento visionario de Nerup en 1990, la tecnología molecular en estudios de interleuquinas (gen-scan y microarrays) han ido aproximando cada vez más a estos mediadores inmunológicos al proceso destructivo de la célula $\beta^{5,21,30-31}$.

Nuestro estudio ha confirmado el potencial papel patogénico que podría tener principalmente IL-1ß. Diversos estudios han demostrado, en forma concluyente, que la IL-1ß puede inducir la expresión de la óxido nítrico-sintetasa (iNOS) y la producción de radicales libres NO, los cuales están capacitados para inhibir la actividad de la aconitasa mitocondrial, suprimir la secreción de insulina y causar daño al ADN, agravando el ambiente $ß$ pancreático ${ }^{32,33}$. Sin embargo, evidencias recientes, en el modelo animal $\mathrm{BB}$, indican que IL-1ß sería una citoquina necesaria, pero no suficiente para completar el proceso de destrucción de la célula $\aleph^{34}$.

Finalmente, se debe señalar que todos los estudios que cuantifican perfiles serológicos, ya sea de citoquinas o anticuerpos circulantes, deben ser interpretados con cautela, puesto que no necesariamente una alteración periférica detectada a nivel plasmático pueda tener un correlato fisiológico exacto con lo que sucede en forma particular en el entorno de la célula $\aleph^{35}$. Esta situación, ha sido incluso evidenciada en nuestros estudios previos de anticuerpos antialbúmina bovina (BSA), donde un alto título de este anticuerpo detectado en el paciente recién diagnosticado puede, incluso, no tener ningún papel esencial en la declinación funcional de la célula $\aleph^{36}$.

Al igual que otros estudios, nuestros datos confirman que ciertas citoquinas, consideradas diabetogénicas, podrían aumentar la antigenicidad incrementando la expresión de autoantígenos. Sin embargo, el efecto más sobresaliente de estas citoquinas, estaría dado por el daño directo que éstas podrían generar en el islote ${ }^{37,38}$. Es probable que en un futuro cercano, estos mediadores sean factores claves a considerar para establecer un diagnóstico en fase de prediabetes, donde aún sea posible modular niveles de expresión y así prevenir, reducir o atenuar el proceso inmunológico que gatilla la destrucción de la célula $ß$. 


\section{REFERENCIAS}

1. DORMAN JS, BunKer CH. HLA-DQ locus of the human leukocyte antigen complex and type 1 diabetes mellitus: a HuGE review. Epidemiol Rev 2000; 22: 218-27.

2. HirschHorn JN. Genetic epidemiology of type 1 diabetes. Pediatr Diabetes 2003; 4: 87-100.

3. Akerblom HK, Vaarala O, Hyoty H, Ilonen J, Knip M. Environmental factors in the etiology of type 1 diabetes. Am J Med Genet 2002; 115: 18-29.

4. Becker DJ, La Porte RE, Lbman I, Pietropaolo M, Dosch HM. Prevention of type 1 diabetes: Is now the time? J Clin Endocrinol Metab 2000; 85: 498506.

5. LERNMARK A. Autoimmune diseases: are markers ready for prediction. J Clin Invest 2001; 108: 10916.

6. Pietropaolo M, Becker DJ. Type 1 diabetes intervention trials. Pediatric Diab 2001; 2: 2-11.

7. Kukreja A, Cost G, Marker J, Zhang C, Sun Z, LunSu K ET AL. Multiple immunoregulatory defects in type 1 diabetes. J Clin Invest 2002; 109: 131-40.

8. Sú́rez-Pinzón WL, Rabinovitch A. Approaches to type 1 diabetes prevention by intervention in cytokine immunoregulatory circuits. Int J Exp Diabetes Res 2001; 2: 3-17.

9. Durinovic-Beшo I, Riedl M, Rosinger S, Maisel N, Kalbacher H, Deeg M et al. Th2 dominance of T helper cell response to preproinsulin in individuals with preclinical type 1 diabetes. Ann N Y Acad Sci 2002; 958: 209-13.

10. Casares S, BRUMEanu TD. Insights into the pathogenesis of type 1 diabetes: a hint for novel immunospecific therapies. Curr Mol Med 2001; 3: 357-78.

11. SCARIM AL, ARnush M. Evidence for the presence of type 1 IL-1 receptors on $\beta$ cells of islets of Langerhans. Biochim Biophys Acta 1997; 1361: 313-20.

12. Rabinovitch A. An update on cytokines in the pathogenesis of insulin-dependent diabetes mellitus. Diab Metab Rev 1998; 14: 129-51.

13. Erbagci AB, Tarakcioglu M, Coskum Y, Sivasli E, NAMIDURU E. Mediators of inflammation in children with type 1 diabetes mellitus: cytokines in type 1 diabetic children. Clin Biochem 2001; 34: 645-50.
14. ZipRIS D, KarNieli E. A single treatment with IL-4 via retrovirally transduced lymphocytes partially protects against diabetes in biobreeding (BB) rats. JOP 2002; 3: 76-82.

15. Tisch R, Wang B, Weaver DJ, Lu B, Bui T, Arthos J ET AL. Antigen-specific mediated suppression of beta cell autoimmunity by plasmid DNA vaccinacion. J Immunol 2001; 166: 2122-32.

16. Tisch R, Wang B, Atkinson MA, Serreze DV, FRIEDLNE R. A glutamic acid decarboxilase 65specific Th2 cell clone immunoregulates autoimmune diabetes in nonobese diabetic mice. J Immunol 2001; 167: 6925-36.

17. Weaver DJ, Liu B, Tisch R. Plasmid DNAs encoding insulin and glutamic acid decarboxilase 65 have distinct effects on the progression of autoimmune diabetes in nonobese diabetic mice. J Immunol 2001; 167: 586-92.

18. Koarada S, Wu Y, Olshansky G, Ridgway WM. Increased nonobese diabetic Th1:Th2 (INFgamma:IL-4) ratio is CD4+ $\mathrm{T}$ cell intrinsic and independent of APC genetic background. J Immunol 2002; 169: 6580-7.

19. Wolfe T, Bot A, Hughes A, Mohrie U, Rodrigo E, JAUME JC ET AL. Endogenous expresion levels of autoantigens influence success or failure DNA immunizations to prevent type 1 diabetes: addition of IL-4 increases safety. Eur J Immunol 2002; 32: 113-21.

20. Halminen M, Juhela S, Vaarala O, Simmell O, Illonen $\mathrm{J}$. Induction of interferon-gamma and IL-4 production by mitogen and specific antigens in peripheral blood lymphocytes of type 1 diabetes patients. Autoimmunity 2001; 34: 1-8.

21. Horiki M, Yamato E, Noso S, Ikegami H, Ogihara T, MIYAZAKI J. High level expresion of interleukin-4 following electroporation-mediated gene transfer accelerates type 1 diabetes in NOD mice. J Autoimmunity 2003; 20: 111-7.

22. Walz M, Overbergh L, Mathieu C, Kolb H, Martin S. A murine interleukin-4-Ig fusion protein regulates the expression of Th1 and Th2-specific cytokines in the pancreas of NOD mice. Horm Metab Res 2002; 34: 561-9.

23. Hill NJ, Van Gunst K, Sarvetnick N. Th1 and Th2 pancreatic inflammation differentially affects homing of islet-reactive CD4 cells in nonobese diabetic mice. J Immunol 2003; 170: 1649-58. 
24. Pérez-Bravo F, Riesco MV, Albala C, Oyarzún A, Santos JL, Carrasco E. Perfil de autoanticuerpos y lactancia materna en pacientes diabéticos tipo 1 . Rev Méd Chile 2001; 129: 611-9.

25. SchrezenMeIR J, Jagla A. Milk and diabetes. J Am Coll Nutr 2000; 19: 176-90.

26. Steinbrenner H, Nguyen T, Wohlrab U, Scherbaum W, SeISLER J. Effect of proinflammatory cytokines on gene expression of the diabetes associated autoantigen IA-2 in INS-1 cells. Endocrinology 2002; 143: 3839-45.

27. Ordovas JM, Qi L, HaiQIng S, MayeR J. Is IL12B a potential genetic target for the prevention of type 1 diabetes through dietary intervention. Nut Rev 2002; 60: 182-6.

28. Kibirige M, Metcalf B, Renuka R, Wilkin TJ. Testing the accelerator hypothesis. Diabetes Care 2003; 26: 2865-70.

29. Ingberg CM, Sarnblad S, Palmer M, Schvarcz E, Berne C, Aman J. Body composition in adolescent girls with type 1 diabetes. Diab Med 2003; 20: 1005-11.

30. LowE WL. The matrix unloaded: implications for cytokine signaling in islets. Endocrinology 2003; 144: 4262-3.

31. Roер BO. The role of T-cells in the pathogenesis of type 1 diabetes: from cause to cure. Diabetologia 2003; 46: 305-21.

32. Chen MC, Schuit F, Eizirik DL. Identification of IL$1 \beta$ induced messenger RNAs in rat pancreatic $\beta$ cells by differential display of messenger RNA. Diabetologia 1999; 42: 1199-203.

33. Ling Z, Van de Casteele M, Eizirik DL, Pipellers DG. Interleukin-1ß induced alteration in a $\beta$ cell phenotype can reduce cellular sensitivity to conditions that cause necrosis but not to cytokine induced apoptosis. Diabetes 2000; 49: 340-5.
34. Walchin G, Augstein P, Schroder D, Kuttier B, KLoting I, HeinKe P ET AL. IL-1beta, IFN gamma and TNF-alpha increase vulnerability of pancreatic beta cell to autoimmune destruction. J Autoimmun 2003; 20: 303-12.

35. von HeRRATH M, Homann D. Introducing baselines for therapeutic use of regulatory $\mathrm{T}$ cells and cytokines in autoimmunity. Trends Immunol 2003; 24: 540-5.

36. Oyarzún A, Santos Jl, Carrasco E, Albala C, Salnas A, PÉrez F. Anticuerpos anti-albúmina bovina (BSA) en niños diabéticos tipo 1 recién diagnosticados y su asociación con lactancia materna y exposición a leche de vaca. Rev Méd Chile 2003; 131: 865-72.

37. John NE, Andersen HU, Fey SJ, Larsen PM, RoepstoFF P, LARSEN MR ET aL. Cytokine or chemically derived nitric oxide alters the expresión of proteins detected by two-dimensional gel electrophoresis in neonatal rat islets of Langerhans. Diabetes 2000; 49: 1819-29.

38. Mayer A, Rharbaoui F, Thivolet C, Orgiazzi J, Madec AM. The relationship between peripheral $\mathrm{T}$ cell reactivity to insulin, clinical remissions and cytokine production in type 1 (insulin-dependent) diabetes mellitus. J Clin Endocrinol Metab 1999; 84: 2419-24.

39. Hanifi-Moghaddam P, Schloot NC, Kappier S, SeissLER J, KolB H. An association of autoantibody status and serum cytokine levels in type 1 diabetes. Diabetes 2003; 52: 1137-42.

Agradecimientos

Los autores agradecen la colaboración de todos los niños y sus familias participantes en este estudio. A los Hospitales San Juan de Dios, San Borja-Arriarán, E González Cortés y a la Fundación de Diabetes Juvenil de Chile. 